\title{
Achievement goals, motivational self-talk, and academic engagement among Chinese students
}

\author{
Cen Wang ${ }^{1} \cdot$ Sungok Serena Shim ${ }^{2} \cdot$ Christopher A. Wolters $^{3}$
}

Received: 21 March 2016/Revised: 8 May 2017/Accepted: 13 June 2017/Published online: 20 June 2017

(C) Education Research Institute, Seoul National University, Seoul, Korea 2017

\begin{abstract}
The present study investigated the mediating role of motivational self-talk strategies (i.e., mastery selftalk, extrinsic self-talk, and efficacy enhancement self-talk) in the relationships between achievement goals and academic engagement (i.e., involvement, withdrawal in the face of difficulties, avoidance of challenges, and disruptive behaviors) among 1096 10th grade students (43\% male) in a selective Chinese high school. Structural equation models showed that total and desirable effects of mastery goals were partially mediated by motivational self-talk strategies. Performance approach goals were maladaptive and only weak partial mediation was found through motivational self-talk strategies. A similar pattern was observed with performance avoidance goals. Mastery self-talk and efficacy enhancement self-talk were related to positive patterns of engagement while extrinsic self-talk was related to maladaptive patterns of engagement. Theoretical, cultural, and practical implications are discussed.
\end{abstract}

Cen Wang

auwang@csu.edu.au

Sungok Serena Shim

sshim@bsu.edu

Christopher A. Wolters

wolters.21@osu.edu

1 Faculty of Arts and Education, School of Teacher Education, Charles Sturt University, Bathurst, NSW 2795, Australia

2 Department of Educational Psychology, Ball State University, Muncie, IN 47306, USA

3 Department of Educational Studies, Walter E. Dennis Learning Center, College of Education \& Human Ecology, The Ohio State University, Columbus, OH 43210, USA
Keywords Achievement goals - Motivational selfregulation strategies - Mastery self-talk - Extrinsic self-talk · Efficacy enhancement self-talk · Academic engagement

In most classrooms, large variability in motivation is apparent. Some students are interested and persist despite difficulties. Others, however, show maladaptive behaviors such as avoiding challenging tasks, or simply giving up in the face of setbacks. Chinese classrooms are no exception. Compared to most of the Western high school education, Chinese high school education is characterized by large class size, rigorous academic standards, and intense competition (Salili 1996). Thus, academic engagement in Chinese education contexts is challenging yet crucial for students' immediate academic achievement and long-term success. Accordingly, it is critical to investigate the factors leading to optimal engagement.

In the present study, we focused on the nature and function of motivational self-talk. When it gets difficult to persist at a task at hand, instead of simply abandoning the work, students may engage in purposeful self-talk to encourage themselves to persist. Motivational self-talk refers to strategic inner talk, aiming to enhance one's own effort and persistence toward goal completion in demotivating situations (McCann and Garcia 1999; Wolters 1999, 2003). Although the role of self-talk has been emphasized from the constructivist view of education (Vygotsky 1987), the exact function of motivational selftalk with respect to engagement has rarely been examined, especially among secondary school students. While investigating the association between various motivational self-talk strategies, we also considered the antecedent of self-talk. Prior research has emphasized achievement goals 
(i.e., different reasons and purposes for academic learning, see Elliot 2005 for a review) as powerful predictors of engagement as well as motivational self-talk (Wolters and Benzon 2013; Wolters and Rosenthal 2000; Wolters et al. 1996). However, previous studies have examined the roles of achievement goals and motivational self-talk rather independently of each other. According to the social-cognitive model of self-regulated learning, students' use of self-regulatory talks is likely to be affected by their overarching goals (Pintrich 2000, 2004). Thus, we have proposed and tested the viability of a process model, in which achievement goals affect students' engagement through triggering different types of motivational self-talk using structural equation modeling.

\section{Achievement goals and academic engagement}

Three distinct achievement goals have been widely examined in the literature: (a) a mastery goal refers to the focus on learning, understanding, and developing competence, (b) a performance approach goal focuses on demonstrating competence and being judged favorably, and (c) a performance avoidance goal is concerned with masking incompetence. When students approach academic situations with different achievement goals, their cognitive, affective, and behavioral processes and outcomes tend to be qualitatively different (Meece et al. 2006).

Mastery goals have been in general consistently leading to higher academic engagement such as adaptive help seeking, persistence, and preference for challenging tasks. In contrast, performance avoidance goals have led to selfhandicapping strategies, disruptive behaviors, and task disengagement (see Elliot 2005 for a review). The role of performance approach goals, however, has not yielded a consistent pattern. Performance approach goals have been related to avoidant help seeking, cheating, disruptive behaviors, and self-handicapping as well as self-regulation (Elliot and McGregor 2001; Shim and Ryan 2005; Zusho et al. 2005).

Achievement motivation research among Chinese students is less compared to the voluminous literature on the same subject in the Western culture. Mastery goals have been related to deep learning approach (the desire for a deeper understanding of the material), deep strategy use, surface learning approach (i.e., getting by with minimum effort expenditure), and higher effort expenditure (Ho and Hau 2008; Ng 2000; Shi et al. 2001). Performance goals have been related to both deep and surface approaches, achieving approach (the desire for high performance), and higher effort expenditure (Ho and Hau 2008; Ng 2000). In addition, mastery goals and performance avoidance goals were positively and negatively related to achievement (Ho and Hau 2008). However, the relationship between performance approach goals and achievement is not straightforward. Ho and Hau (2008) found performance approach goals to be related to higher math achievement, whereas Chan (2008) found that performance approach goals did not predict achievement in any of the three areas: academic, non-academic, or social/leadership areas. In general, the patterns of the relationships between achievement goals and Chinese students' educational outcomes are not different from those conducted in the Western culture. That is, mastery goals are adaptive, whereas performance avoidance goals are maladaptive. Performance approach goals, however, have mixed results and fail to yield a consistent pattern of relations. Conceptually, mastery goals and performance avoidance goals are expected to have direct positive and negative effects, respectively, on academic engagement. However, due to the inconsistent findings in the literature, we do not form a hypothesis for performance approach goals.

\section{Self-talk as motivational self-regulatory strategy}

To date, only a few studies have examined how motivational self-regulation strategies might affect academic engagement. These strategies can take various forms but all are exercised to initiate and maintain motivation or to control motivational processing for optimal performance (Pintrich 2004; Wolters 2003). Among the many types of motivational self-regulatory strategies, we focus on motivational self-talk. Although motivational self-talk has rarely been studied in educational settings, the function of self-talk (private speech or inner speech in Vygotsky's terminology) more generally has been emphasized in Vygotsky's early work (1987). Meichenbaum (1977) also has studied self-talk as a strategy for behavioral control in young children. In the present study, we examined three different types of motivational self-talk: mastery self-talk, performancelextrinsic self-talk (referred to as extrinsic self-talk hereafter for brevity), and efficacy enhancement self-talk. These motivational self-talk strategies have a clear focus on the regulation of motivational beliefs (e.g., achievement goals, self-efficacy) and involve students' efforts to remind themselves of their reasons for completing a task or to reassure themselves of their ability to complete a task successfully in order to achieve effort enhancement and persistence in demotivating situations. These strategies have been examined in a handful of previous studies (Hatzigeorgiadis 2006; Wolters 1998; Wolters and Benzon 2013) but the effects of these various strategies remained unclear.

Mastery self-talk refers to the type of self-talk in which students remind themselves of the personal importance of 
maximum learning and mastery of the task/skills (Wolters 1998, 2003). Extrinsic self-talk refers to the type of selftalk reminding oneself of the importance of getting good grades or other extrinsic outcomes (Wolters 1998, 2003). Both types of self-talk are goal oriented but emphasize different desired end-results. Efficacy enhancement selftalk aims to enhance individuals' self-efficacy to successfully learn the material even if it is challenging (McCann and Garcia 1999). For instance, students may tell themselves "You are doing a good job. You will be successful if you just keep at it." (Wolters 2003).

Research showed that using a variety of motivational self-regulation strategies may enhance effort and performance (Schwinger et al. 2009; Schwinger and StiensmeierPelster 2012). When high school and university students were categorized into different groups depending on their profiles of strategy use (Schwinger et al. 2012), the group of students who scored low on all motivational self-regulation strategies exerted the least effort compared to those that scored high on all strategies, which showed the highest effort expenditure. Similarly, in study 2, Schwinger et al. (2012) reported that the group of university students who were high on all motivation self-regulation strategies showed the highest effort and performance followed by the goal-focused group of students who scored high on mastery self-talk, extrinsic self-talk, and performance avoidance self-talk (i.e., reminding oneself of a potential failure). However, the group of students who scored high on only extrinsic self-talk and performance avoidance self-talk (i.e., a performance self-talk profile) showed the lowest effort and performance level, together with the group low on all motivational self-regulation strategies.

The fact that the performance self-talk group scored lower than the goal-focused group on effort and performance seemed to indicate the adaptive nature of mastery self-talk which buffered the maladaptive effect of performance self-talk. Supporting this argument were the reported positive links between mastery self-talk and planning, monitoring, and effort in other studies (Schwinger and Stiensmeier-Pelster 2012; Wolters 1999). However, the role of extrinsic self-talk in motivation regulation processes remains unclear. In fact, extrinsic self-talk has been not only linked to higher effort (Schwinger and StiensmeierPelster 2012), higher metacognition, and better course grades (Wolters 1999) but also showed null relationship with effort (Wolters 1999) and grade (Schwinger and Stiensmeier-Pelster 2012). In addition to such inconsistent findings, no empirical studies have been conducted with Chinese students.

Efficacy enhancement self-talk has been examined often in the sport domain and has been consistently linked to desirable outcomes such as enhanced performance, effort, and higher self-efficacy beliefs (Hatzigeorgiadis 2006;
Latinjak et al. 2011; Zinsser et al. 2006). To date, no research has examined how this type of self-talk influences outcomes in the academic context. McCann and Garcia (1999) developed a self-report measure for volitional strategies among which is efficacy enhancement. However, the effect of this construct (i.e., criterion validity) was not fully investigated in this study. Wolters (1998) examined the role of efficacy enhancement in motivational regulation but considered efficacy enhancement through students' effort to make the task easier so that they could feel more competent. Thus, in Wolters (1998) study, the level of efficacy was not controlled by self-talk, rather through enhancing the possibility of successfully completing the task. In sum, we do not know whether this strategy is more or less effective as compared to other types.

Based on the limited evidence, we are not able to form reliable hypotheses. However, conceptually, we speculate that all three types of motivational self-talk strategies will be related to a desirable pattern of academic engagement (e.g., high involvement and/or low withdrawal in the face of difficulties, avoidance of challenge, and disruptive behaviors) because using some kind of strategy should be better than not using at all. In addition, given the conceptually unclear nature of extrinsic goals (Sansone and Harackiewicz 2000), we expect that the effect of extrinsic self-talk might be weak or null.

\section{The process model linking achievement goals, motivational self-talk, and engagement}

According to Pintrich (2000, 2004), self-regulated learning is likely to serve as a mediator of the effects of overarching goals on learning outcomes. Conceptually, achievement goals are the overarching cognitive representation of competence striving (Elliot 2005), whereas motivational self-talk strategies are individuals' inner control of their thoughts and students' engagement are externally manifested behaviors. Social cognitive model of self-regulation (Pintrich 2000) proposed four phases for motivational selfregulation, namely, forethought, planning, and activation phase; monitoring phase; control and regulation phase; and reaction and reflection phase. Theoretically, students' motivational beliefs activated in the activation phase are likely to serve as the antecedents of students' choice of motivational self-regulation strategies in the control phase as individuals explicitly and implicitly pursue congruence between the motivational belief such as achievement goals and the means chosen to attain the set goal (Wolters and Benzon 2013; Wolters and Rosenthal 2000). This sequence is also consistent with Zimmerman's model of self-regulation process (Zimmerman and Campillo 2003, p. 239), as motivational self-regulation aims to regulate the elements 
during Forethought phase (e.g., goal orientations). In turn, motivational self-regulation which deliberately targets achieving optimal motivation for goal completion, serves as a theoretical prerequisite for the explicit manifestations of academic engagement (Zimmerman 1985).

To date, there are studies that examined the associations between achievement goals and motivational self-talk or the links between motivational self-talk and outcomes. However, there has been no direct test of mediational processes of motivational self-talk. For example, two extant studies have examined the relationships between achievement goals and motivational self-talk (Wolters and Benzon 2013; Wolters and Rosenthal 2000). Both studies have found that mastery goals positively predicted mastery self-talk, whereas performance approach goals positively predicted extrinsic self-talk. However, the link between mastery goals and extrinsic self-talk was not entirely clear, as it was found in one study (Wolters and Rosenthal 2000) but not in another (Wolters and Benzon 2013). Thus, theoretically, the association between mastery goals and mastery self-talk is expected but the scarce empirical studies also reported the link between mastery goals and extrinsic self-talk. To date, nothing is known about the link between achievement goals and efficacy enhancement selftalk.

Various cognitive (e.g., view of effort and failure) and affective processes (e.g., affective attitude toward school work) are likely to mediate the link between achievement goals and engagement (Elliot 2005). Thus, we do not believe that motivational self-talk is the only or the strongest mediator of the effects of achievement goals on learning outcomes. As we consider motivational self-talk as one of the many mediators of the effects of achievement goals, we expect partial mediation models where achievement goals will show direct effects on outcomes even after accounting for self-talk. However, due to the lack of empirical data and inconsistencies in the existing data, we do not make specific hypotheses of the mediated effects.

\section{The significance of the present study}

This study explores three research questions: (a) how do achievement goals predict academic engagement? (b) how does motivational self-talk relate to academic engagement? and (c) how does motivational self-talk mediate the relations between achievement goals and academic engagement? The present study makes three unique contributions to the lean literature on motivational self-regulation. First, we investigate the viability of the mediation model in which motivational self-talk, as one type of self-regulated learning strategies, mediates the relations between achievement goals and academic engagement. With this overall aim, the present study also adds evidence to the links between achievement goals and three forms of selftalk, and between these forms of self-talk and four indicators of students' academic engagement.

Secondly, given that motivational self-regulation is defined as students' active regulation of motivation for enhanced effort and persistence to achieve goal completion (Wolters 2003), investigation of indicators of lack of quality engagement (i.e., avoidance of challenges; disruptive behaviors) or the opposite of persistence (e.g., withdrawal in the face of difficulties) will be useful to obtain a fuller picture of the nature and effectiveness of motivational self-regulation strategies. Most studies on this topic focused on the positive indicators of engagement (e.g., planning, monitoring, effort, performance). In addition, the variables such as withdrawal in the face of difficulties and avoidance of challenge are expected to be particularly responsive to motivational self-regulation, as one may not need to regulate motivation while working on easy tasks.

Thirdly, our sample of Chinese secondary students provides an interesting and unique context to study motivational self-talk. The few existing studies of motivational self-talk have examined only Western samples. In China, high school graduates are required to take the competitive and high stake test of National College Entrance Examination. The students in our sample attend a selective high school, and thus, they are even more pressured to do well and get admitted into prestigious colleges. Although these students are highly motivated to do well, there is likely to be individual variability in students' engagement at school. It is imperative to engage in effective motivation regulation to handle such high demands and pressure (Salili 1996).

\section{Method}

\section{Participants and procedure}

This research was conducted in a large high school (serving 10th-12th grade) in an industrial city in the Northeastern part of China. The school is highly selective, admitting the top $16 \%$ of the middle school graduates in the city. All 10th grade students $(N=1182)$ received a package containing a parent consent form and a self-report survey. They were instructed to return the completed materials in a sealed envelope to their teachers the following Monday. The survey was completed anonymously. The response rate was $93 \%$ and a total of 109610 th graders $(43 \%$ male and $57 \%$ female; mean age $=16.8$ years; $\mathrm{SD}=.69$ ) returned completed surveys. 


\section{Measures}

For the 50-item self-report survey, students responded to five-point Likert-type scales regarding their general achievement goals, motivational self-talk, and academic engagement. Response choices ranged from Not at all true of me (1) to Very true of me (5). The original items were in English and therefore needed to be translated into Chinese. To ensure validity of the translated version of the measures, we followed a standard translation and back-translation procedure for this process (see Hambleton and Patsula 1998, and van de Vijver and Tanzer 2004 for more information).

\section{Achievement goals}

Three types of achievement goals were measured using items from the Patterns of Adaptive Learning Survey (PALS; Midgley et al. 2000), capturing students' purposes in academic learning in school in general. The mastery goals scale assessed students' focus on learning, understanding, and developing competence (5 items; $\alpha=.81$; e.g., It is important to me that I thoroughly understand my class work). The performance approach goals scale measured students' focus on demonstrating and validating their competence (5 items; $\alpha=.85$; e.g., One of my goals is to look smart in comparison to the other students in my class). The performance avoidance goals scale assessed students' focus on masking incompetence (4 items; $\alpha=.77$; e.g., It is important to me that I do not look stupid in class).

\section{Motivational self-talk}

Three types of motivational self-talk were measured. Mastery self-talk refers to students' self-reported use of self-talk that highlights the importance of learning and mastery (Wolters et al. 2005; 6 items; $\alpha=.85$; e.g., I tell myself that I should keep working just to learn as much as I can). Extrinsic self-talk refers to students' reported use of self-talk that highlights the importance of getting good grades (Wolters et al. 2005; 5 items; $\alpha=.88$; e.g., I remind myself about how important it is to get good grades). $E f$ ficacy enhancement self-talk refers to students' reported use of self-talk that reassures their level of perceived competence (McCann and Garcia 1999; 5 items; $\alpha=.82$; e.g., I tell myself "I can do this").

\section{Academic engagement}

Academic engagement included involvement (Eccles 1983), withdrawal in the face of difficulties (Midgley et al.
1993), avoidance of challenges (Midgley et al. 1993), and disruptive behaviors (PALS; Midgley et al. 2000). Involvement measured students' beliefs about how hard they work for school ( 7 items; $\alpha=.84$; e.g., I am engaged when doing homework). Withdrawal refers to students' self-reported behaviors of quitting when they are doing difficult academic work (4 items; $\alpha=.81$; e.g., When I do not understand my work, I give up and do something else). Avoidance of challenge refers to students' self-reported behaviors of skipping or avoiding challenging academic work (4 items; $\alpha=.81$; e.g., I would avoid difficult class work whenever possible). Disruptive behaviors capture students' behaviors that annoy the teacher, break classroom rules, and disturb the lesson (5 items; $\alpha=.86$; e.g., I sometimes disturb the lesson that is going on in class).

\section{Results}

Means, standard deviations, zero-order correlations, and reliability coefficients (i.e., Cronbach's $\alpha$ ) among variables are shown in Table 1. All our analyses were conducted using Mplus version 7 (Muthén and Muthén 2012). To determine model fit, we followed $\mathrm{Hu}$ and Bentler's (1999) recommendation: Comparative Fit Index $(\mathrm{CFI})>.90$, Root Mean Square Error of Approximation (RMSEA) <.08, Standardized Root Mean Square Residual (SRMR) $<.08$, and Tucker-Lewis Index (TLI) $>.90$ are considered to have an adequate model fit.

\section{Results from structural equation modeling}

Before we ran our main analyses, we conducted confirmatory factor analyses on our variables. The 3-factor structure of motivational self-talk strategies was adequate fit to the data, $\chi^{2}(86)=387.51, \chi^{2} / \mathrm{df}=4.51, \mathrm{RMSEA}=.06 ; \mathrm{CFI}=.94$, $\mathrm{TLI}=.92, \mathrm{SRMR}=.04$, with the standardized item factor loadings ranging from .62 to .82 . The 4 -factor structure model for academic engagement was good fit to the data, $\chi^{2}(161)=483.24, \chi^{2} / \mathrm{df}=3.00$, RMSEA $=.04 ;$ CFI $=$ $.95, \mathrm{TLI}=.95, \mathrm{SRMR}=.05$, with the standardized item factor loadings ranging from .47 to .82 . Unlike the CFA models of motivational self-talk and academic engagement (reported in the measurement section), CFA with all three factors of achievement goals resulted in a non-positive definite covariance matrix. An inspection of the correlations between latent variables indicated that the correlation was greater than 1 (1.004) between latent variables of performance approach and avoidance goals, as suggested by high correlation between these two observed variables $(r=.83)$. This suggests that the performance approach and avoidance 


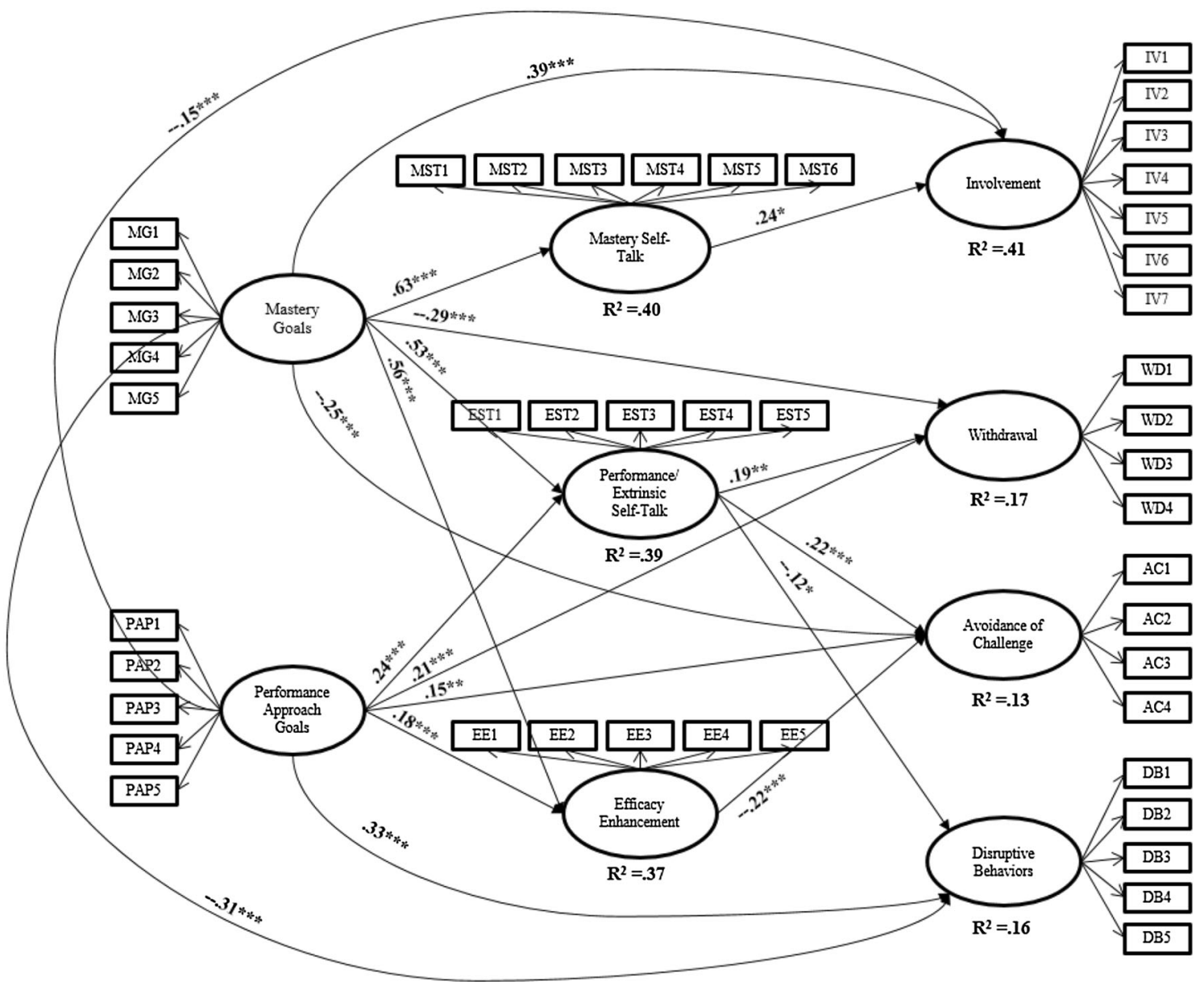

Fig. 1 This is the mediation model between mastery goals and performance approach goals and outcomes. Only significant paths are shown and all path coefficients are standardized. $* p<.05$, ** $p<.01$, *** $p<.001$

goals could not be distinguished which resulted in an inadmissible model.

Thus, we compared the option of combining two performance goals vs. running the model with each type of performance goals at a time. When we ran the model with mastery goals and combined performance goals (approach goals and avoidance goals), the overall model was not good fit to the data, $\chi^{2} / \mathrm{df}=7.40$, RMSEA $=.08, \mathrm{CFI}=.92$, $\mathrm{TLI}=.89, \mathrm{SRMR}=.07$. This model also had higher AIC and BIC values ${ }^{1}$, compared to the 2 -factor models with mastery goals and one of the performance goals, which yielded adequate fit to the data, $\chi^{2} / \mathrm{dfs}<5.56$, RMSEAs $\leq .07$, CFIs $>.95$, TLIs $>.92$, SRMRs $\leq .06$, with the standardized item factor loadings ranging from .50 to .80 . In addition to the poor fit to the data, it is not recommended to combine performance approach and

\footnotetext{
1 Akaike Information Criterion (AIC) and Bayesian Information Criterion (BIC) are general fit statistics for both nested and nonnested models. They measure unexplained variance between the predicted and observed models. Both of them penalizes for the lack of parsimony. Smaller AIC and BIC are considered to be desirable.
}

avoidance goals considering the theoretical and empirical support of the distinction between performance approach and avoidance goals (see Linnenbrink-Garcia et al. 2012 for discussion on this matter). Thus, we decided to run our analyses with each type of performance goals at a time. The results of both models were mostly identical and thus, we present the model with performance approach goals (Fig. 1) and only provide the model with performance avoidance goals in Fig. 2 for brevity. Our approach will enable comparison to other studies, which utilized the trichotomous framework of achievement goals (i.e., mastery goals, performance approach goals, performance avoidance goals) (Linnenbrink-Garcia et al. 2008).

Structural equation modeling was conducted using bootstrapping with a resampling of 1000 to test the significance of the indirect effects of achievement goals and students' academic engagement. Bootstrapping is one of the most valid approaches with high power to detect intervening variable effect (Preacher and Hayes 2008). The error terms of the self-talk strategies, the error terms of academic engagement, and the covariance between the two 


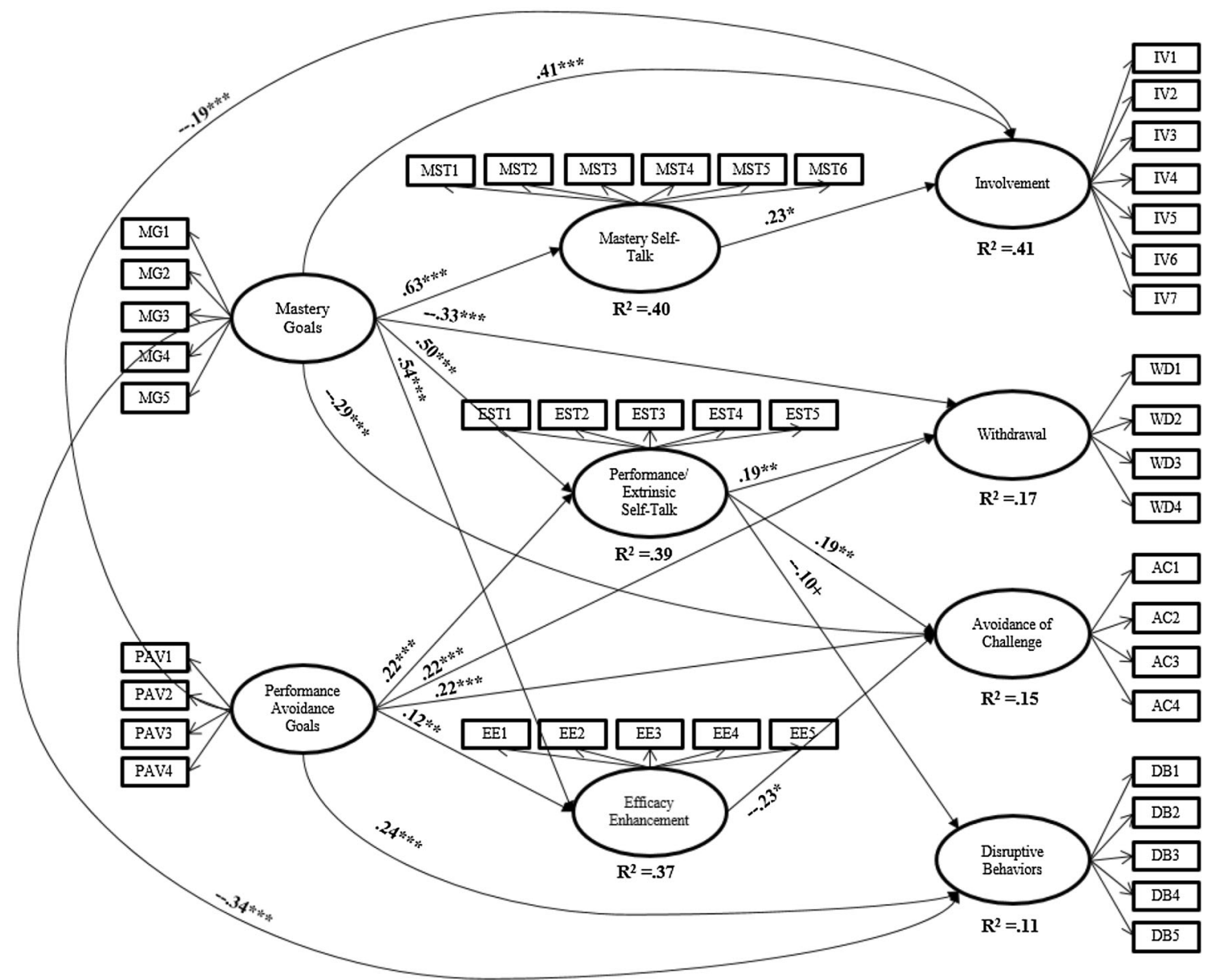

Fig. 2 This is the mediation model between mastery goals and performance avoidance goals and outcomes. Only significant paths are shown and all path coefficients are standardized. ${ }^{+} p<.06, * p<.05, * * p<.01, * * * p<.001$

types of achievement goals were included in the model. Overall, the structural equation model was adequate fit to the data, $\chi^{2}(940)=2525.98, \chi^{2} / \mathrm{df}=2.69$, RMSEA $=$ $.04, \mathrm{CFI}=.93, \mathrm{TLI}=.93, \mathrm{SRMR}=.05$. As shown in Fig. 1, a mastery goal was positively related to all three forms of motivational self-talk $\left(\beta_{\mathrm{s}} \geq .53, p<.001\right)$ and the magnitudes of the standardized path coefficients were larger compared to those of a performance approach goal. A performance approach goal was related to extrinsic selftalk $(\beta=.24, p<.001)$ and efficacy enhancement selftalk $(\beta=.18, p<.001)$ but unlike a mastery goal, was not related to mastery self-talk.

We expected that using motivational self-talk would be beneficial, at least to some extent, regardless of its type. The prediction held true for mastery self-talk and efficacy enhancement self-talk but not for extrinsic self-talk. Mastery self-talk was related to high involvement $(\beta=.24$, $p=.02)$. Similarly, efficacy enhancement self-talk was related to lower avoidance of challenges $(\beta=-.22$, $p=.04)$. The pattern associated with extrinsic self-talk was unclear. It was associated with not only increased withdrawal $(\beta=.19, p<.01)$ and avoidance of challenges $(\beta=.22, p<.001)$ but also lower disruptive behaviors $(\beta=-.12, p=.02)$.

\section{Indirect effects between achievement goals and academic engagement}

As shown in Tables 2 and 3, a mastery goal was linked to a desirable profile of students' academic engagement. Specifically, a mastery goal was related to higher involvement $(\beta=.59, p<.001)$, but lower withdrawal $(\beta=-.34, p<.001)$, avoidance of challenges $(\beta=-.29$, $p<.001)$ and disruptive behaviors $(\beta=-.29, p<.001)$. A performance approach goal, however, was linked to a negative profile of students' academic engagement, such as lower involvement $(\beta=-.13, p<.001)$, higher withdrawal $(\beta=.23, p<.001)$, avoidance of challenges $(\beta=.16, p<.001)$, and disruptive behaviors $(\beta=.30$, $p<.001)$. 
Table 1 Means, standard deviations, correlations, and reliability among variables $(N=1096)$

\begin{tabular}{|c|c|c|c|c|c|c|c|c|c|c|c|}
\hline & & 1 & 2 & 3 & 4 & 5 & 6 & 7 & 8 & 9 & 10 \\
\hline 1 & Mastery goals & & & & & & & & & & \\
\hline 2 & Performance approach goals & $.15 * *$ & & & & & & & & & \\
\hline 3 & Performance avoidance goals & $.21 * *$ & $.83 * *$ & & & & & & & & \\
\hline 4 & Mastery self-talk & $.52 * *$ & $.12 * *$ & $.12 * *$ & & & & & & & \\
\hline 5 & Performance/extrinsic self-talk & $.48 * *$ & $.31 * *$ & $.31 * *$ & $.61 * *$ & & & & & & \\
\hline 6 & Efficacy enhancement & $.47 * *$ & $.23 * *$ & $.22 * *$ & $.70 * *$ & $.58 * *$ & & & & & \\
\hline 7 & Involvement & $.48 * *$ & -.01 & -.01 & $.46 * *$ & $.33 * *$ & $.40 * *$ & & & & \\
\hline 8 & Withdrawal & $-.27 *^{*}$ & $.12 * *$ & $.12 * *$ & $-.20 * *$ & $-.07 *$ & $-.19 * *$ & $-.39 * *$ & & & \\
\hline 9 & Avoidance of challenges & $-.21 * *$ & $.09 * *$ & $.11 * *$ & $-.18 * *$ & -.03 & $-.18 * *$ & $-.32 * *$ & $.78 * *$ & & \\
\hline \multirow[t]{4}{*}{10} & Disruptive behaviors & $-.22 * *$ & $.20 * *$ & $.14 * *$ & $-.11 * *$ & $-.09 * *$ & $-.07 * *$ & $-.31 * *$ & $.38 * *$ & $.31 * *$ & \\
\hline & Mean & 3.91 & 2.75 & 2.97 & 3.10 & 3.35 & 3.16 & 3.46 & 2.27 & 2.58 & 1.68 \\
\hline & Standard deviation & .79 & .98 & .95 & .84 & .90 & .84 & .71 & .83 & .86 & .73 \\
\hline & Reliability (Cronbach's alpha) & .81 & .85 & .77 & .85 & .88 & .82 & .84 & .81 & .81 & .85 \\
\hline
\end{tabular}

$* p<.05, * * p<.01$

With regard to indirect effects, mastery self-talk partially mediated the effects of a mastery goal on involvement (indirect effect $\beta=.15,95 \%$ CI $[.02, .27]$, accounting for $25 \%$ of the total effects) while efficacy enhancement self-talk partially mediated its effect on avoidance of challenges (indirect effect $\beta=-.12,95 \% \mathrm{CI}$ $[-.002,-.24]$, accounting for $41 \%$ of the total effects). A mastery goal was also linked to the use of extrinsic selftalk, which had mixed relations with academic engagement.

Extrinsic self-talk partially mediated the effects of performance approach goals on withdrawal and avoidance of challenges: A performance approach goal was related to extrinsic self-talk, which related to higher withdrawal (indirect effect $\beta=.05,95 \%$ CI [.02, .08], accounting for $22 \%$ of the total effects) and avoidance of challenges (indirect effect $\beta=.05,95 \%$ CI $[.02, .09]$, accounting for $31 \%$ of the total effects).

\section{Discussion}

The guiding premise of the present investigation was that different achievement goals may lead students to utilize different forms of self-talk, which in turn affect their academic engagement. We aimed to investigate whether certain type of achievement goals predisposes students to employ self-talk of a different nature. In addition, we were particularly interested to find out whether all forms of motivational self-talk were associated with enhanced academic engagement and if so, whether some types of selftalk were more closely linked than others to students' academic engagement. The present data generated several intriguing patterns that may bear important implications for researchers and educators.

\section{Research question 1: how do achievement goals predict academic engagement?}

A mastery goal was positively related to involvement, but negatively related to withdrawal, avoidance of challenges, and disruptive behaviors. This finding is similar to a number of previous studies showing the adaptive function of mastery goals. However, performance approach goals were consistently linked to negative functioning such as higher withdrawal, avoidance of challenges, disruptive behaviors, and lower involvement. In prior studies, the effects of performance approach goals have been less consistent. Accordingly, there has been much debate among researchers about the nature and the function of performance approach goals (see Harackiewicz et al. 2002; Midgley et al. 2001). This pattern of consistent negative effect of performance approach goals can be related to the fact that performance approach and avoidance goals fail to form separate factors. A higher correlation between the two types of performance goals has often been seen among Asian samples (Murayama and Elliot 2009; Tao and Hong 2000; Zusho et al. 2005). This pattern might be related to the Collectivistic feature of Chinese culture, which emphasizes the importance of filial piety, obedience, and respect for parents and other authority figures (Salili and Lai 2003). For Asian students, academic success is an important avenue for one to "fulfill their filial obligation" (Fuligni and Zhang 2004). This high filial obligation may, at the same time, trigger a fear of failure of losing face for the families (Steinberg et al. 1992). Such a dynamic may 
Table 2 Total, direct, and indirect effects between mastery goals and outcomes with motivational self-talk as mediators

\begin{tabular}{|c|c|c|c|c|c|}
\hline \multirow[t]{2}{*}{ Paths } & \multirow[t]{2}{*}{ Total effects } & \multirow[t]{2}{*}{ Direct effects } & \multicolumn{3}{|l|}{ Indirect effects } \\
\hline & & & \multicolumn{3}{|l|}{$95 \% \mathrm{CI}$} \\
\hline \multirow[t]{4}{*}{ Mastery goals $\rightarrow$ involvement } & \multirow[t]{4}{*}{$.59 * * *$} & \multirow[t]{4}{*}{$.39 * * *$} & \multicolumn{3}{|c|}{$.20 * * *[.14, .26]$} \\
\hline & & & Thru mas_ST & Thru perf_ST & Thru EFC \\
\hline & & & $.15^{*}$ & $-.01(\mathrm{~ns})$ & $.06(\mathrm{~ns})$ \\
\hline & & & {$[.02, .27]$} & {$[-.07, .05]$} & {$[-.06, .17]$} \\
\hline \multirow[t]{4}{*}{ Mastery goals $\rightarrow$ withdrawal } & \multirow[t]{4}{*}{$-.34 * * *$} & \multirow[t]{4}{*}{$-.29 * * *$} & \multicolumn{3}{|c|}{$-.05(\mathrm{~ns})[-.13, .03]$} \\
\hline & & & Thru mas_ST & Thru perf_ST & Thru EFC \\
\hline & & & $-.06(\mathrm{~ns})$ & $.10 * *$ & $-.09(\mathrm{~ns})$ \\
\hline & & & {$[-.20, .08]$} & {$[.04, .17]$} & {$[-.21, .03]$} \\
\hline \multirow[t]{4}{*}{ Mastery goals $\rightarrow$ avoidance of challenges } & \multirow[t]{4}{*}{$-.29 * * *$} & \multirow[t]{4}{*}{$-.25 * * *$} & \multicolumn{3}{|c|}{$-.04(\mathrm{~ns})[-.12, .05]$} \\
\hline & & & Thru mas_ST & Thru perf_ST & Thru EFC \\
\hline & & & $-.03(\mathrm{~ns})$ & $.12 * * *$ & $-.12 *$ \\
\hline & & & {$[-.18, .12]$} & {$[.05, .19]$} & {$[-.24,-.002]$} \\
\hline \multirow[t]{4}{*}{ Mastery goals $\rightarrow$ disruptive behavior } & \multirow[t]{4}{*}{$-.29 * * *$} & \multirow[t]{4}{*}{$-.31 * * *$} & \multicolumn{3}{|c|}{$.02(\mathrm{~ns})[-.05, .08]$} \\
\hline & & & Thru mas_ST & Thru perf_ST & Thru EFC \\
\hline & & & .09 (ns) & $-.07 *$ & -.01 \\
\hline & & & {$[-.04, .23]$} & {$[-.12,-.01]$} & {$[-.12, .10]$} \\
\hline
\end{tabular}

Due to rounding, the total effects may not necessarily be the sum of indirect and direct effects Mas_ST mastery self-talk, Perf_ST performance/extrinsic self-talk, EFC efficacy enhancement $* p<.05, * * p<.01, * * * p<.001$

Table 3 Total, direct, and indirect effects between performance approach goals and outcomes with motivational self-talk as mediators

\begin{tabular}{|c|c|c|c|c|c|}
\hline \multirow[t]{2}{*}{ Paths } & \multirow[t]{2}{*}{ Total effects } & \multirow[t]{2}{*}{ Direct effects } & \multicolumn{3}{|l|}{ Indirect effects } \\
\hline & & & \multicolumn{3}{|l|}{$95 \% \mathrm{CI}$} \\
\hline \multirow[t]{4}{*}{ Performance approach goals $\rightarrow$ involvement } & \multirow[t]{4}{*}{$-.13 * * *$} & \multirow[t]{4}{*}{$-.15^{* * * *}$} & \multicolumn{3}{|c|}{$.02(\mathrm{~ns})[-.03, .07]$} \\
\hline & & & Thru mas_ST & Thru perf_ST & Thru EFC \\
\hline & & & $.01(\mathrm{~ns})$ & $-.01(\mathrm{~ns})$ & $.02(\mathrm{~ns})$ \\
\hline & & & {$[-.02, .03]$} & {$[-.03, .02]$} & {$[-.02, .06]$} \\
\hline \multirow[t]{4}{*}{ Performance approach goals $\rightarrow$ withdrawal } & \multirow[t]{4}{*}{$.23 * * *$} & \multirow[t]{4}{*}{$.21 * * *$} & \multicolumn{3}{|c|}{$.02(\mathrm{~ns})[-.03, .06]$} \\
\hline & & & Thru mas_ST & Thru perf_ST & Thru EFC \\
\hline & & & $.00(\mathrm{~ns})$ & $.05^{* *}$ & $-.03(\mathrm{~ns})$ \\
\hline & & & {$[-.02, .01]$} & {$[.02, .08]$} & {$[-.07, .01]$} \\
\hline \multirow[t]{4}{*}{ Performance approach goals $\rightarrow$ avoidance of challenges } & \multirow[t]{4}{*}{$.16^{* * *}$} & \multirow[t]{4}{*}{$.15 * *$} & \multicolumn{3}{|c|}{$.01(\mathrm{~ns})[-.03, .05]$} \\
\hline & & & Thru mas_ST & Thru perf_ST & Thru EFC \\
\hline & & & $-.00(\mathrm{~ns})$ & $.05 * *$ & $-.04(\mathrm{~ns})$ \\
\hline & & & {$[-.01, .01]$} & {$[.02, .09]$} & {$[-.08, .004]$} \\
\hline \multirow[t]{4}{*}{ Performance approach goals $\rightarrow$ disruptive behavior } & \multirow[t]{4}{*}{$.30 * * *$} & \multirow[t]{4}{*}{$.33 * * *$} & \multicolumn{3}{|c|}{$-.03 * * *[-.07, .01]$} \\
\hline & & & Thru mas_ST & Thru perf_ST & Thru EFC \\
\hline & & & $.00(\mathrm{~ns})$ & $-.03 *$ & $-.00(\mathrm{~ns})$ \\
\hline & & & {$[-.01, .02]$} & {$[-.06,-.002]$} & {$[-.04, .03]$} \\
\hline
\end{tabular}

Due to rounding, the total effects may not necessarily be the sum of indirect and direct effects Mas_ST mastery self-talk, Perf_ST performance/extrinsic self-talk, EFC efficacy enhancement $* p<.05, * * p<.01, * * * p<.001$ 
also explain high adoption of performance avoidance goals (Chen and Stevenson 1995). Such a pattern is also often observed within Asian samples (Murayama and Elliot 2009). Similar to a prior study that found the highest fear of failure among many high achieving Asian American students (Steinberg et al. 1992), high-ability students in our sample are not an exception to the trend either. To date, there is no clear and strong evidence for the commonly observed moderate to high correlation between performance approach and avoidance goals to be the function of culture (Linnenbrink-Garcia et al. 2012). We speculate that high correlation between the two performance goals in our study may be related to the unique interaction between the Collectivist culture and the competitive learning environment among high achieving peers.

\section{Research question 2: how does motivational self-talk relate to academic engagement?}

Among three types of self-talk, performance/extreme selftalk showed significant relations with three engagement outcomes while mastery and efficacy enhancement selftalk were related to one engagement outcome each. Mastery self-talk and efficacy enhancement self-talk were associated with heightened academic engagement. However, the present study showed a mixed picture of extrinsic self-talk, which was linked to maladaptive outcomes, such as higher withdrawal, and avoidance of challenges as well as adaptive outcomes such as lower disruptive behaviors. This is consistent with the previous research which reported positive functions of extrinsic self-talk in terms of effort, metacognition, and grades (Schwinger and Stiensmeier-Pelster 2012; Wolters 1999), but null relations with a maladaptive outcome such as procrastination in a previous study (Wolters and Benzon 2013). We speculated that in an educational and cultural milieu that emphasizes performance, reminding oneself of the importance of achieving superior performance may be effective in orienting students away from classroom rule breaking or disturbing the lesson underway. However, ultimately, this strategy may make the stakes of failure more salient and trigger the tendency to withdraw and avoid challenges, as challenging tasks often require students to embrace a higher risk of failure.

Despite the association with withdrawal and avoidance of challenges, extrinsic self-talk was reportedly used most frequently among our participants. Such frequent use of extrinsic self-talk may not be surprising given the competitive educational context in China (Salili 1996) and students' filial piety to meet parents' expectations for high achievement. However, unfortunately, the phenomenon does not seem to be unique to Chinese students; extrinsic self-talk was also rated as the most identified strategy among American and German students (Schwinger et al. 2012; Wolters and Benzon 2013). Taken together, while extrinsic self-talk may yield immediate benefit (e.g., reducing disruptive behaviors, short-term grades), this strategy may compromise students' long-term academic success by hampering the tendency to seek challenge and persevere in the face of failure, which are critical for longterm success. Future research with longitudinal design can test the viability of such a possibility.

\section{Research question 3: how does motivational self-talk mediate the relations between achievement goals and academic engagement?}

Not all of the mediation links were found to be significant, as expected. Students reported adoption of mastery goals was related positively to all three types of self-talk, albeit with different intensities. Mastery goal pursuit seems to lead students to utilize all available motivational strategies regardless of the type. This pattern was also found in Wolters and Rosenthal (2000). It is encouraging to find that mastery goals predicted mastery self-talk and efficacy enhancement self-talk, which were linked to important yet different engagement outcomes. However, special attention should be given to the link between mastery goals and extrinsic self-talk. Even though mastery goal-oriented students may use all kinds of available strategies with good intentions (i.e., the desire to learn and master), they should be aware of the caveat that extrinsic self-talk is linked to undesirable behaviors such as withdrawal and avoidance of challenges.

Participants' reported focus on performance approach goals was related to extrinsic self-talk as well as efficacy enhancement self-talk. The path coefficients were generally much weaker when compared to those between mastery goals and self-talk. It seems natural that performance goaloriented students reminded themselves of the very end-state of their goal: doing better than others and achieving good grades. The engagement outcomes, if this path is taken, will be playing safe (i.e., higher withdrawal in the face of difficulties and avoidance of challenge) and not causing trouble in class (i.e., low disruptive behaviors), which is unlikely to contribute to long-term academic growth.

The link between performance approach goals and efficacy enhancement self-talk was also significant. To accomplish their goal of outperforming others or getting good grades, they may need to keep boosting their efficacy to combat the anxiety or frustration. Important to note is that efficacy enhancement self-talk is linked to lower challenge avoidance but it does not lead to involvement. While it is important to seek challenges, hard work is a 
necessary condition to master schoolwork. Performance approach goals did not predict mastery self-talk, which was the only significant predictor of involvement. More importantly, this goal type had direct negative relations with involvement. Taken together, performance approach goals may promote the use of some types of motivational strategies but the engagement outcomes driven by this goal type are limited to say the least.

Under the current Chinese education system with such a salient emphasis on performance approach goals (Tao and Hong 2000), it might be challenging to dissuade teachers and parents from emphasizing performance approach goals. However, it is important to inform them the potential cost associated with promoting such goals. Instead of trying to eliminate performance approach goals, teachers and parents can focus on promoting and modeling mastery goals. Research on parental achievement goals (Friedel et al. 2007) and parenting styles (Gonzalez et al. 2002) provides practical tips for promoting desirable forms of achievement goals among students. While doing so, as we emphasized above, mastery goal-oriented students should be coached and scaffolded to focus on desirable types of self-talk. Mastery goal-oriented students are not completely immune to maladaptive motivational self-talk strategies.

\section{Limitations and future directions}

The current study utilized self-report instruments to tap into students' achievement goals, use of motivational selftalk, and their level of academic engagement. Our instruments are vulnerable to some types of bias (e.g., response bias such as social desirability, inaccurate memory, cognitive distortions). Self-report provides insights into individuals' perceptions of themselves and their behaviors and such perceptions shape actual behaviors (Crick and Dodge 1994). However, for academic engagement behaviors, other data collection methods such as teacher ratings and observations can be used in the future studies.

Given that achievement goals and motivational selfregulatory strategies exert influence on each other (Ning and Downing 2010), future extension of the current study can use longitudinal designs or developmental cascade designs to address the issue of directionality as well as to provide more rigorous test of mediation processes (Masten and Cicchetti 2010). Considering the link between academic engagement and actual achievement has yet to be established, the inclusion of school grade or other objective indicators of academic achievement should be considered in the future research. In our study, we could not fit the performance approach and avoidance goals within the same model due to their high correlation.
Additional research that replicates the current study is needed before we draw further conclusions whether this pattern is unique to our sample or common among all Asian students.

\section{Conclusion}

The present findings are especially important for highperforming Asian students who work under pressure in a competitive environment. In such an environment, students are likely to endorse performance approach goals and may resort to extrinsic self-talk, which we found to be mostly maladaptive. The present results also indicate that the ability to monitor one's motivational state and implement appropriate motivational self-talk strategies has important implications for academic engagement. However, not all types of self-talk are equally beneficial. The present study highlights the importance of approaching academic tasks with a desirable goal focus. However, it is important to note that having the right goal focus does not guarantee the utilization of adaptive types of self-talk. Highly mastery goal-focused students also need support and guidance to utilize adaptive self-talk strategies.

\section{References}

Chan, D. W. (2008). Goal orientations and achievement among Chinese gifted students in Hong Kong. High Ability Studies, 19, 37-51. doi:10.1080/13598130801980307.

Chen, C., \& Stevenson, H. W. (1995). Motivation and mathematics achievement: A comparative study of Asian-American, CaucasianAmerican, and East Asian high school students. Child Development, 66, 1215-1234. doi:10.1111/j.1467-8624.1995.tb00932.x.

Crick, N. R., \& Dodge, K. A. (1994). A review and reformulation of social-information-processing mechanisms in children's social adjustment. Psychological Bulletin, 115, 74-101. doi:10.1037/ 0033-2909.115.1.74.

Eccles, J. (1983). Expectancies, values, and academic behaviors. In J. T. Spence (Ed.), Achievement and achievement motives: Psychological and sociological approaches (pp. 75-146). San Francisco: Freeman.

Elliot, A. J. (2005). A conceptual history of the achievement goal construct. In A. J. Elliot \& C. S. Dweck (Eds.), Handbook of competence and motivation (pp. 52-72). New York: Guilford Press.

Elliot, A. J., \& McGregor, H. A. (2001). A $2 * 2$ Achievement goal framework. Journal of Educational Psychology, 80, 501-519. doi:10.1037/0022-3514.80.3.501.

Friedel, J. M., Cortina, K. S., Turner, J. C., \& Midgley, C. (2007). Achievement goals, efficacy beliefs and coping strategies in mathematics: The roles of perceived parent and teacher goal emphases. Contemporary Educational Psychology, 32, 434-458. doi:10.1016/j.cedpsych.2006.10.009.

Fuligni, A. J., \& Zhang, W. (2004). Attitudes toward family obligation among adolescents in contemporary urban and rural China. Child Development, 74, 180-192. doi:10.1111/j.14678624.2004.00662.x. 
Gonzalez, A. R., Doan Holbein, M. F., \& Quilter, S. (2002). High school students' goal orientations and their relationship to perceived parenting styles. Contemporary Educational Psychology, 27, 450-470. doi:10.1006/ceps.2001.1104.

Hambleton, R. K., \& Patsula, L. (1998). Adapting tests for use in multiple languages and cultures. Social Indicators Research, 45, 153-171. doi:10.1023/A:1006941729637.

Harackiewicz, J. M., Barron, K. E., Pintrich, P. R., Elliot, A. J., \& Thrash, T. M. (2002). Revision of achievement goal theory: Necessary and illuminating. Journal of Educational Psychology, 94, 638-645. doi:10.1037/0022-0663.94.3.638.

Hatzigeorgiadis, A. (2006). Instructional and motivational self-talk: An investigation on perceived self- talk functions. Hellenic Journal of Psychology, 3, 164-175.

Ho, I. T., \& Hau, K. T. (2008). Academic achievement in the Chinese context: The role of goals, strategies, and effort. International Journal of Psychology, 43, 892-897. doi:10.1080/ 00207590701836323.

Hu, L., \& Bentler, P. M. (1999). Cutoff criteria for fit indexes in covariance structure analysis: Conventional criteria versus new alternatives. Structural Equation Modeling, 6, 1-55. doi:10. 1080/10705519909540118.

Latinjak, A. T., Torregrosa, M., \& Renom, J. (2011). Combining selftalk and performance feedback: Their effectiveness with adult tennis players. The Sport Psychologist, 25, 18-31.

Linnenbrink-Garcia, L., Middleton, M. J., Ciani, K. D., Easter, M. A., O'Keefe, P. A., \& Zusho, A. (2012). The strengths of the relation between performance-approach and performance-avoidance goal orientations: Theoretical, methodological, and instructional implications. Educational Psychologist, 47, 281-301. doi:10. 1080/00461520.2012.722515.

Linnenbrink-Garcia, E. A., Tyson, D. F., \& Patall, E. A. (2008). When are achievement goal orientation beneficial for academic achievement? A closer look at main effects and moderating factors. International Review of Social Psychology, 21, 19-70.

Masten, A. S., \& Cicchetti, D. (2010). Developmental cascades. Development and Psychopathology, 22, 491-495. doi:10.1017/ S0954579410000222.

McCann, E. J., \& Garcia, T. (1999). Maintaining motivation and regulating emotion: Measuring individual differences in academic volitional strategies. Learning and Individual Differences, 11, 259-279. doi:10.1016/S1041-6080(99)80003-X

Meece, J. L., Anderman, E. M., \& Anderman, L. H. (2006). Classroom goal structure, student motivation and academic achievement. Annual Review of Psychology, 57, 487-503. doi:10.1146/annurev.psych.56.091103.070258.

Meichenbaum, D. H. (1977). Cognitive behavior modification: An integrative approach. New York: Plenum Press.

Midgley, C., Kaplan, A., \& Middleton, M. (2001). Performance approach goals: Good for what, for whom, under what circumstances, and at what cost? Journal of Educational Psychology, 93, 77-86. doi:10.1037/0022-0663.93.1.77.

Midgley, C., Maehr, M. L., Hruda, L. Z., Anderman, E., Anderman, L., Freeman, K. E., et al. (2000). Manual for the patterns of adaptive learning scales. Ann Arbor: University of Michigan.

Midgley, C., Maehr, M. L., \& Urdan, T. (1993). Patterns of adaptive learning survey (PALS). Ann Arbor: University of Michigan.

Murayama, K., \& Elliot, A. J. (2009). The joint influence of personal achievement goals and classroom goal structures on achievement-relevant outcomes. Journal of Educational Psychology, 101, 432-447. doi:10.1037/a0014221.

Muthén, L. K., \& Muthén, B. O. (2012). Mplus user's guide (7th ed.). CA: Los Angeles.

Ng, C. H. (2000). A path analysis of self-schema, goal orientation, learning approaches and performance. Journal of Psychology in Chinese Societies., 1, 93-121.
Ning, H. K., \& Downing, K. (2010). The reciprocal relationship between motivation and self-regulation: A longitudinal study on academic performance. Learning and Individual Differences, 20, 682-686. doi:10.1016/j.lindif.2010.09.010.

Pintrich, P. R. (2000). The role of goal orientation in self-regulated learning. In M. Boekaerts, P. Pintrich, \& M. Zeidner (Eds.), Handbook of self-regulation (pp. 451-502). San Diego: Academic Press.

Pintrich, P. R. (2004). A conceptual framework for assessing motivation and self-regulated learning in college students. Educational Psychology Review, 16, 385-407. doi:10.1007/ s10648-004-0006-x.

Preacher, K. J., \& Hayes, A. F. (2008). Asymptotic and resampling strategies for assessing and comparing indirect effects in multiple mediator models. Behavior Research Methods, 40, 879-891. doi:10.3758/BRM.40.3.879.

Salili, F. (1996). Accepting personal responsibility for learning. In D. A. Watkins \& J. B. Biggs (Eds.), The Chinese learner: Cultural, psychological and contextual influences (pp. 85-105). Hong Kong: The Central Printing Press, CERC and ACER.

Salili, F., \& Lai, M. K. (2003). Learning and motivation of Chinese students in Hong Kong: A longitudinal study of contextual influences on students' achievement orientation and performance. Psychology in the schools, 40, 51-70. doi:10.1002/pits. 10069.

Sansone, C., \& Harackiewicz, J. M. (2000). Looking beyond rewards: The problem and promise of intrinsic motivation. In C. Sansone \& J. M. Harackiewicz (Eds.), Intrinsic and extrinsic motivation: The search for optimal motivation and performance. San Diego, CA: Academic Press.

Schwinger, M., Steinmayr, R., \& Spinath, B. (2009). How do motivational regulation strategies affect achievement: Mediated by effort management and moderated by intelligence. Learning and Individual Differences, 19, 621-627. doi:10.1016/j.lindif. 2009.08.006.

Schwinger, M., Steinmayr, R., \& Spinath, B. (2012). Not all roads lead to Rome-Comparing different types of motivational regulation profiles. Learning and Individual Differences, 22, 269-279. doi:10.1016/j.lindif.2011.12.006.

Schwinger, M., \& Stiensmeier-Pelster, J. (2012). Effects of motivation regulation on effort and achievement: A mediation model. International Journal of Educational Research, 56, 35-47. doi:10.1016/j.ijer.2012.07.005.

Shi, K., Wang, P., Wang, W., Zuo, Y., Liu, D., Maehr, M. L., et al. (2001). Goals and motivation of Chinese students-Testing the adaptive learning model. In F. Salili, C. Y. Chiu, \& Y. Y. Hong (Eds.), Student motivation: The culture and context of learning (pp. 249-270). New York: Kluwer Academic.

Shim, S., \& Ryan, A. (2005). Changes in self-efficacy, challenge avoidance, and intrinsic value in response to grades: The role of achievement goals. The Journal of Experimental Education, 74, 333-349. doi:10.3200/JEXE.73.4.333-349.

Steinberg, L., Dornbusch, S. M., \& Brown, B. B. (1992). Ethnic differences in adolescent achievement: An ecological perspective. American Psychologist, 47, 723-729. doi:10.1037/0003066X.47.6.723.

Tao, V., \& Hong, Y. Y. (2000). A meaning system approach to Chinese students' achievement goals. Journal of Psychology in Chinese Societies, 1, 13-38.

van de Vijver, F., \& Tanzer, N. K. (2004). Bias and equivalence in cross-cultural assessment: An overview. Revue européenne de psychologie appliquée, 54, 119-135.

Vygotsky, L. S. (1987). Thinking and speech (N. Minick, Trans.). In R. W. Rieber \& A. S. Carton (Eds.), The collected works of L.S. Vygotsky. Problems of general psychology (Vol. 1, pp. 37-285). New York: Plenum (Original work published 1934). 
Wolters, C. (1998). Self-regulated learning and college students' regulation of motivation. Journal of Educational Psychology, 90, 224-235. doi:10.1037/0022-0663.90.2.224.

Wolters, C. (1999). The relation between high school students' motivational regulation and their use of learning strategies, effort, and classroom performance. Learning and Individual Differences, 11, 281-299. doi:10.1016/S1041-6080(99)80004-1.

Wolters, C. (2003). Regulation of motivation: Evaluating an underemphasized aspect of self- regulated learning. Educational Psychologist, 38, 189-205. doi:10.1207/S15326985EP3804_1.

Wolters, C., \& Benzon, M. (2013). Assessing and predicting college students' use of strategies for the self-regulation of motivation. The Journal of Experimental Education, 81, 199-221. doi:10. 1080/00220973.2012.699901.

Wolters, C., Pintrich, P., \& Karabenick, S. (2005). Assessing academic self-regulated learning. In K. Moore \& L. Lippman (Eds.), What do children need to flourish?: Conceptualizing and measuring indicators of positive development (pp. 251-270). New York, NY: Springer.

Wolters, C., \& Rosenthal, H. (2000). The relation between students' motivational beliefs and attitudes and their use of motivational regulation strategies. International Journal of Educational Research, 33, 801-820. doi:10.1016/S0883-0355(00)00051-3.

Wolters, C., Yu, S., \& Pintrich, P. R. (1996). The relation between goal orientation and students' motivational beliefs and selfregulated learning. Learning and Individual Differences, 8, 211-238.

Zimmerman, B. (1985). The development of "intrinsic" motivation: A social learning analysis. Annals of Child Development, 2, 117-160.

Zimmerman, B. J., \& Campillo, M. (2003). Motivating self-regulated problem solvers. In J. E. Davidson \& R. Sternberg (Eds.), The nature of problem solving. New York: Cambridge University Press.

Zinsser, N., Bunker, L., \& Williams, J. (2006). Cognitive techniques for building confidence and enhancing performance. In J. M. Williams (Ed.), Applied sport psychology: Personal growth to peak performance (5th ed., pp. 349-381). New York: McGraw Hill.

Zusho, A., Pintrich, P. R., \& Cortina, K. S. (2005). Motives, goals, and adaptive patterns of performance in Asian American and Anglo American students. Learning and Individual Differences, 15, 141-158. doi:10.1016/j.lindif.2004.11.003. 